\title{
Opto-electronic Emulation of a Programmable Digital Circuit
}

\author{
E. E. Rodríguez*, H. J. Zúñiga*, M. L. Calvo ${ }^{+}$, E. Tepichín* \\ * National Institute for Astrophysics, Optics and Electronics, Erro \#1, Tonantzintla, 72000, Pue. México \\ Faculty of Physical Sciences, Complutense University of Madrid, C. Universitaria, 28040, Mad. España
}

\begin{abstract}
A programmable digital circuit typically consists of a microprocessor, a memory device, an input data device and an output data device, with its corresponding communication busses. These devices work following a program which is stored in the memory device, as codified instructions. An opto-electronic system that emulates a programmable digital circuit is proposed and described in this work. This circuit is commanded by a sequential program stored as a spatial distribution of Fourier Holograms into a photorefractive crystal. The sequential program is conformed by instructions, which have been codified as binary pixels maps. To read and decode each instruction, the spatial position of the photorefractive crystal must change; and then a CCD camera captures the output image of the Fourier Hologram. Our LabVIEW software decodes the instruction from the captured output image and it commands the cireuit to realize the decoded instruction. This software controls the CCD camera and an electromechanical system which moves the spatial position of the photorefractive crystal. The electromechanical system is continuously in communication with a $\mathrm{PC}$ through our software. This is because the electronic circuit of the electromechanical system, moves three stepper motors; depending on the position in which the crystal needs to be to read the next instruction. The experimental results and the complete description of the practical advantages and disadvantages of the complete engineering system and the emulation of the opto-electronic programmable digital circuit will be described in this work.
\end{abstract}

\section{INTRODUCTION.}

The Programmable Digital Circuit (PDC) is an electronic system, with a task that is commanded by instructions that are following a sequential program. The PDC is the main part of many electronic digital circuits, for instance the found ones in computers, measurement instruments, cellular phones, calculators, etc [1]. We describe in this work an electro-optical system which works as a PDC does. To have the best understanding, this work was planned in the next way. The next section concerns the main parts of a typical PDC, and what are its functions. Then our proposal for the experimental setup for an Opto-electronic Programmable Digital Circuit (OePDC) is detailed in the third section. An example of the implementation of our OePDC is described in the fourth section and finally its experimental characteristics are analyzed and studied.

\section{PROGRAMMABLE DIGITAL CIRCUIT.}

The simplest PDC is physically composed by a microprocessor, a memory device, an input data device and an output data device; which are known as the PDC hardware. These devices are controlled by instructions stored in the memory; these instructions are the main part of the PDC software.

\subsection{Hardware.}

Fig. 1 shows a schematic diagram of the simplest PDC hardware. The microprocessor is the most important component in a PDC; it controls the memory, the input data device, and the output data device through connections called busses. The main microprocessor tasks in a PDC are: the data transfer between it and the others devices, and the execution of logic and arithmetic operations. Table 1 includes a list of the most common logic and arithmetic microprocessor operations [2]. The numeric data needed to realize the next logic or arithmetic operation, the actual instruction code, and the data result of the last operation, are stored in the main memory device into the 
microprocessor chip. But the instructions to be executed by the microprocessor are stored into an external device known as auxiliary memory; which in this work is going to be emulated by a photorefractive crystal device.

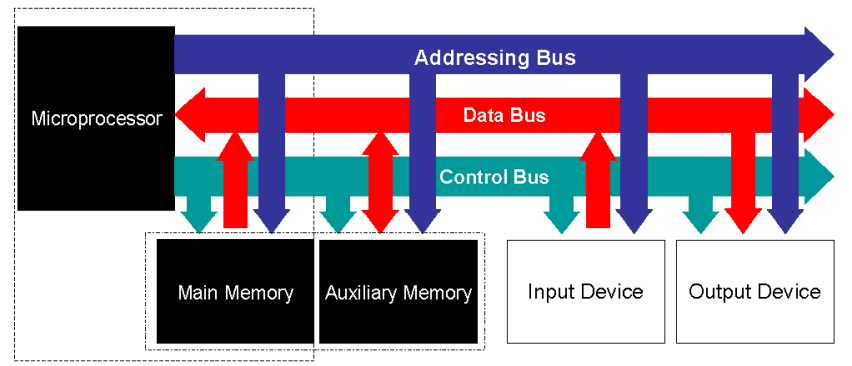

Figure 1. Schematic diagram of a Programmable Digital Circuit

Table 1. Logic and arithmetic microprocessor operations.

\begin{tabular}{|ll|ll|ll|}
\hline OPERATIONS & DESCRIPIION & OPERA'IONS & DESCRIP'IION & OPERATIONS & DESCRIPIION \\
Addition & Arithmetic & Division & Arithmetic & OR & Logic Addition \\
Subtraction & Arithmetic & NEG & Arithmetic inversion & AND & Logic multiplication \\
Multiplication & Arithmetic & & & NOT & Logic inversion \\
\hline
\end{tabular}

\subsection{Software.}

As mentioned before, the PDC software is a sequential program which consists of instructions. Each instruction is executed in a clock cycle. During a clock cycle four tasks are realized. The first task consists in searching for an instruction in the auxiliary memory unit. The second task is the microprocessor interpretation of this instruction. The third task consists in reading data from a memory address, if required. Finally, the fourth task is the logic or arithmetic operation execution by the microprocessor. The main instructions used in PDC software are described in Table $2[3]$.

Table 2. Basic PDC instructions.

\section{EXPERIMENTAL SETUP FOR AN OPTO-ELECTRONIC PROGRAMMABLE DIGITAL CIRCUIT.}

Fig. 2a shows the schematic representation of the experimental setup of our proposal for an OePDC; it consists of a Single Lens Holographic Memory (SLHM) which as mentioned before works as the auxiliary memory, and a Mechatronic System, which is going to be used as the rest of the PDC hardware.

\subsection{Single Lens Holographic Memory.}

The laser beam is divided into the reference and object beams. The object beam is expanded and spatially filtered, to have a divergent spherical wavefront without high frequency noise. This object beam is modulated at the information input plane by a Spatial Light Modulator (SLM). In this case the SLM is a Liquid Crystal Display (LCD), which displays the information in the form of a 2-D distribution of "on" and "off" pixels, which we call in this work an Information Binary Page (IBP binary pixel maps). The implement LCD is a VGA screen with $640 \times 480$ pixels of resolution. The recording element of our system is a $1 \mathrm{~cm}^{3}$ cubic $\mathrm{LiNbO}_{3}$ photorefractive crystal. Note that the single-lens produces simultaneously both the Fourier plane, located into the photorefractive crystal and the output plane $[4,5]$. Therefore the light distribution at the holographic plane is proportional to the Fourier transform of the 
IBP displayed in the SLM; that is, we are storing IBP Fourier Holograms (IFH). The reconstructed image, at the output plane is then captured by a Charger Coupled Device (CCD) camera and sends it back to the PC for feedback purposes. Because we are using IFH whose physical dimensions are of the order of $0.5 \mathrm{~mm}$, we can store (in principle) several holograms into the crystal, if we slightly displaced it.

\subsection{Mechatronic System.}

We have implemented a three dimensional position mechanism which is essentially based on the displacement of three micrometric screws (see Fig. 3a). To each one of these screws a bipolar stepper motor with an angular resolution of 1.8 degrees was coupled, giving an equivalent linear resolution of $0.02 \mathrm{~mm}$ for the $\mathrm{X}, \mathrm{Y}$ and $\mathrm{Z}$ axes. Because the average diameter of the diffraction pattern is around $0.5 \mathrm{~mm}$, this linear resolution is enough to have the minimal displacement necessary to store different IFH without interference. To take advantage of our crystal the information storage in form of IHF was distributed in XY Information Pages (IP) as is shown schematically in Fig. $3 \mathrm{~b}$. To have a high diffraction efficiency, the angle between the reference and object beams $(\theta)$ has to be equal to 13 degrees; Fig. 3c shows the spatial distribution of the IP into the photorefractive crystal. Considering the spatial distribution of the IP and the Bragg angle, is possible to store as much as $182 \mathrm{IFH}$ in our prototype.

The stepper motors are controlled with the electronic circuit showed in Figs. $3 \mathrm{~d}$ and 3e, which is the interface between the mechanism and a computer. The electronic circuit design is protected for mechanical overloads; because each one of the stepper motor controllers has its own power supply. This electronic circuit is commanded by a microcontroller; which was programmed by a Proportional Integral Position Digital Control Algorithm [5b]. The retro-signals are the voltages between the potentiometers terminals; these voltages values are proportional to the positions of each one of the three micrometric screws.

In addition, the electronic circuit maintains an uninterrupted communication with the computer by mean of a Virtual Instrument (VI) in LabVIEW ${ }^{\mathbb{B}}$. The frontal panel of the VI in $\mathrm{LabVIEW}^{\mathbb{B}}$ has a control designated to change continuously the crystal position (Fig. 4a).

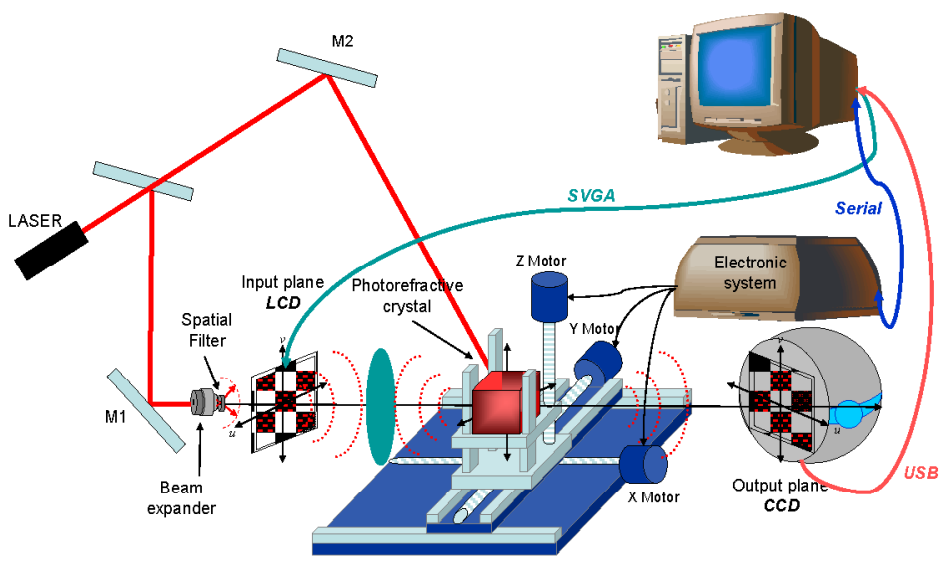

a)

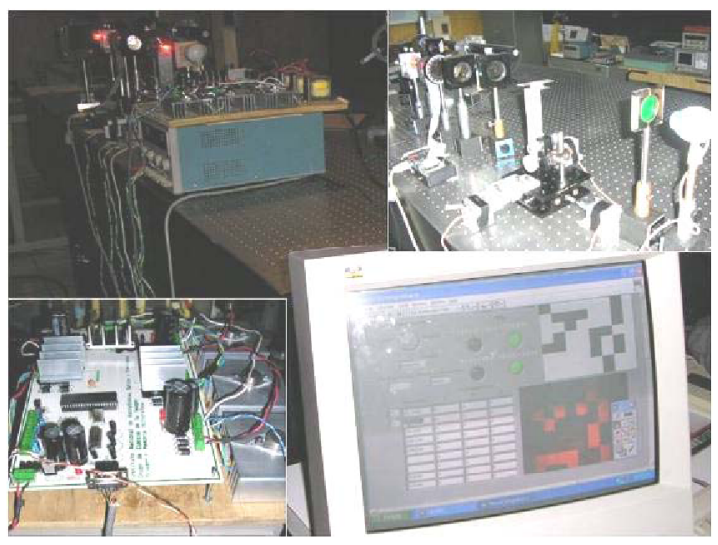

b)

Figure 2. a) Schematic setup for the Opto-electronic Programmable Digital Circuit. b) Picture of the experimental selup.

\section{EXAMPLE OF THE EXPERIMENTAL IMPLEMENTATION FOR AN OPTO-ELECTRONIC PROGRAMMABLE DIGITAL CIRCUIT.}

In our OePDC experimental implementation the input device is the computer keyboard whereas the output device is the computer monitor. Our SLHM takes the auxiliary memory unit function; this is, to store the sequence program as IFH. The holographic single-lens imaging system is our data bus, whereas our addressing bus is the spatial position of the photorefractive crystal. Our computer emulates the microprocessor through the processing LabVIEW $^{\circledR}$ program. 


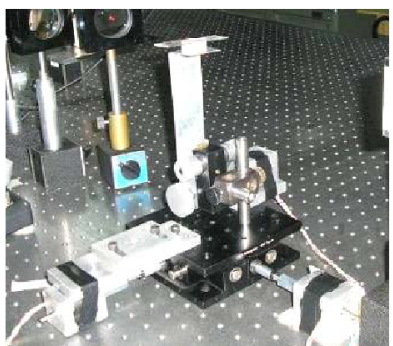

a)

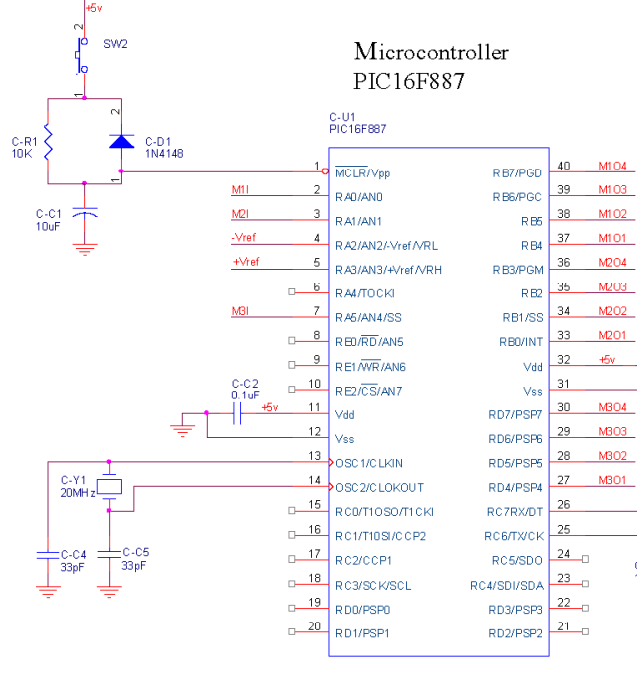

d)

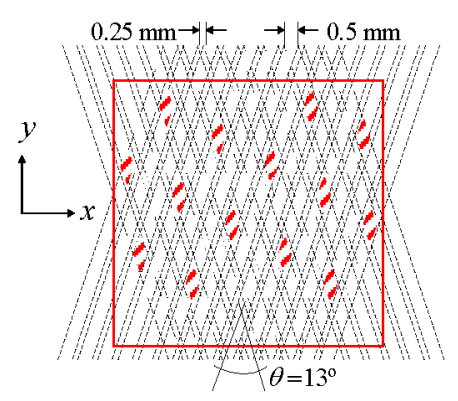

b)

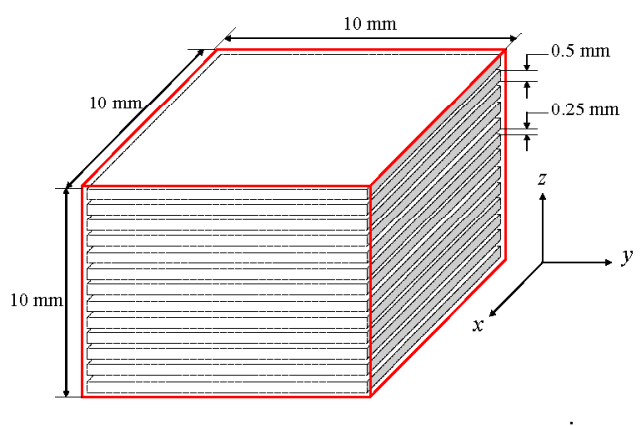

c)

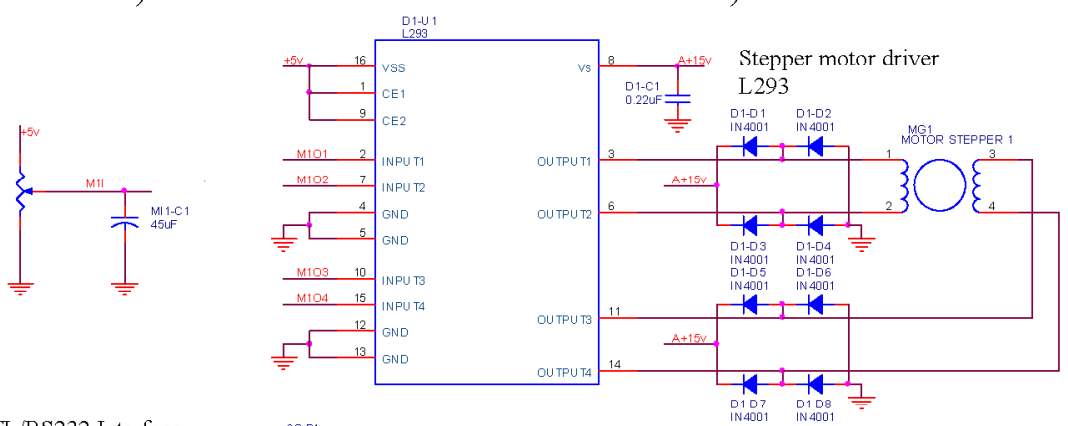

TTL/RS232 Interface
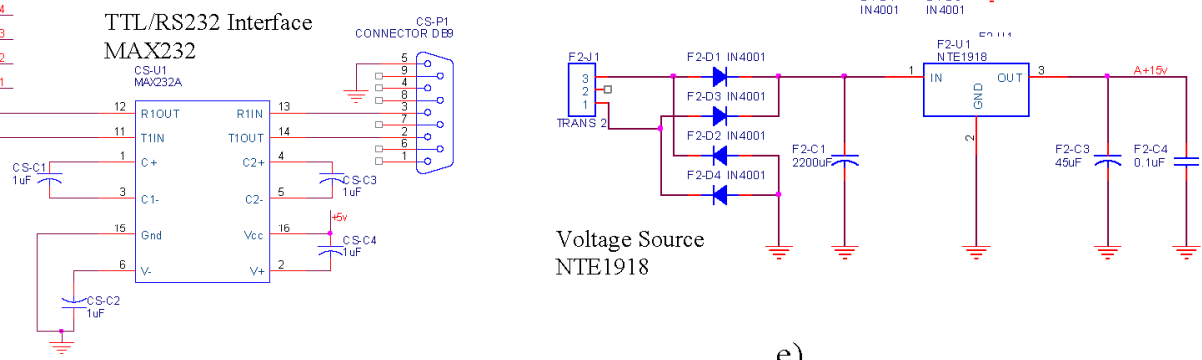

NTE1918

e)

Figure 3. a) Mechanism for the 3D displacement of the crystal, b) Spatial distribution of the IHF into an IP, c) Spatial distribution of the IP into the crystal, d) Microcontroller and communication part, e) Stepper motor controller and retro-signal.

The program to be stored in the SLHM is described in Table 3. The holographic memory stores the information as IFH, which are the IBP Fourier holograms; so we have to codify the proposed program in its corresponding IBP. Table 4 , shows the binary codification of each program instruction. All instructions were codified as a 16 bits binary code. The information distribution in an $\mathrm{HBP}$ is described by Fig. $4 \mathrm{~b}$. As we can see, two instructions and its 16 bits data (if they are needed) are codified in each IBP. In the program sequence the first instruction in the IBP is the first to be realized, and if the logic sequence requires it, the program continues with the second one. The IBP for each couple of instructions and its IFH spatial localizations in the photorefractive crystal are shown in Fig. 4d and Table 5 respectively

\subsection{Processing LabVIEW ${ }^{\circledR}$ Software.}

The processing LabVIEW ${ }^{\circledR}$ software, was developed to select between two options. The first option is the information writing. If the user has selected this option, the program begins displaying an IBP in the LCD. This IBP is the codification of the first of all the instructions previously written, in the control table (Fig. 4c). In this table the user writes the spatial coordinate in which it is desired to store the IFH into the crystal. After the time delay needed to store the hologram, the crystal is moved by the $3 \mathrm{D}$ displacement mechanism, to the position in which it is going to be stored the next IFH. Then this sequence is followed, until the last IFH has been stored. As was described, the IFH stored into the crystal are the Fourier holograms of the IBP of the program instructions listed in Table 3. 
Table 3. List of instructions for the proposed program

\begin{tabular}{|lll|lll|lll|}
\hline & INT. & DESCRIPTION & & INT. & DESCRIPTION & & INT. & DESCRIPTION \\
1 & CLA & Clear $A C$ (register) & 7 & AND D2 & Logic $A C=A C+D 2$ & 13 & IOF & Stop the program \\
2 & LDA 00 & Load 00 in $A C$ & 8 & SPA & Jump the next if $A C>0$ & 14 & LDA 03 & Load 03 in $A C$ \\
3 & AND D2 & Logic $A C=A C+D 2$ & 9 & IOF & Stop the program & 15 & AND D2 & Logic $A C=A C+D 2$ \\
4 & SPA & Jump the next if $A C>0$ & 10 & LDA 02 & Load 02 in $A C$ & 16 & SPA & Jump the next if $A C>0$ \\
5 & IOF & Stop the program & 11 & AND D2 & Logic $A C=A C+D 2$ & 17 & IOF & Stop the program \\
6 & LDA 01 & Load 01 in $A C$ & 12 & SPA & Jump the next if $A C>0$ & 18 & BUN & Return to the 2 int. \\
\hline
\end{tabular}

Table 4. Binary codification of the instructions of the program.

\begin{tabular}{|c|c|c|c|c|c|}
\hline INT. & CODIFICATION & INT. & CODIFICATION & INT & CODIFICATION \\
\hline C.T.A & & SPA & & T.DA & \\
\hline $\mathrm{IOF}$ & & AND & & BUN & \\
\hline
\end{tabular}

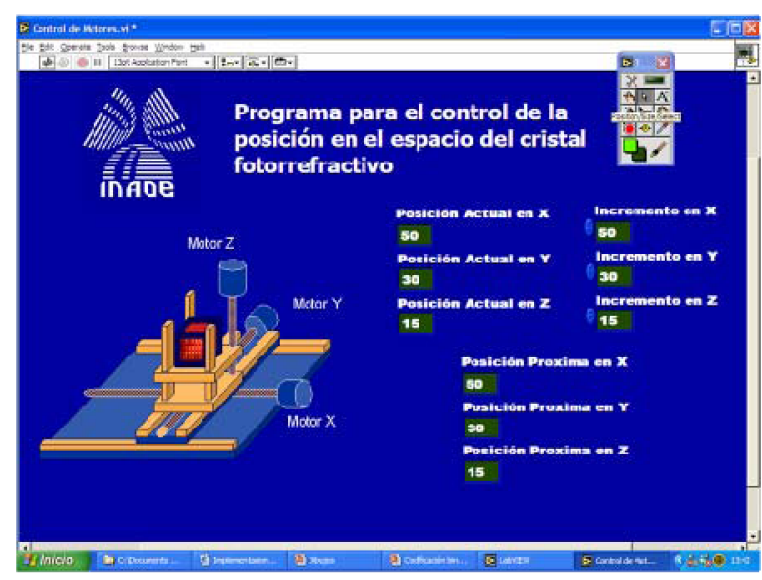

a)

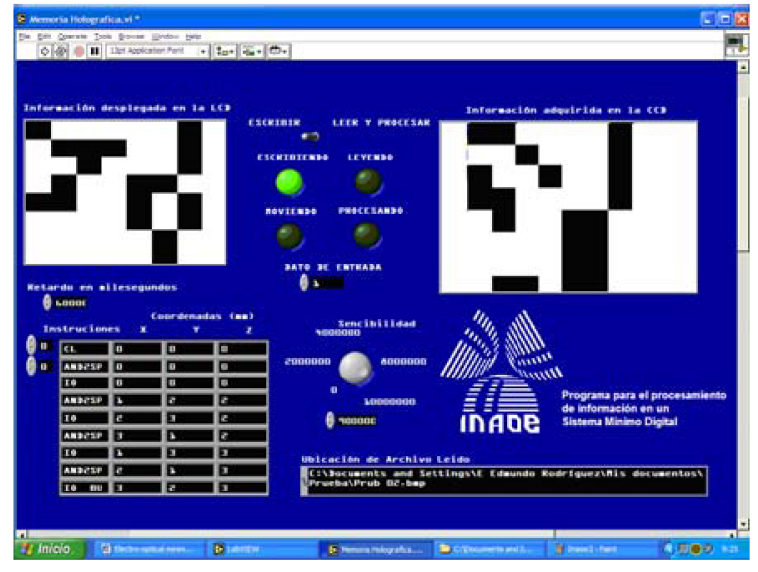

c)
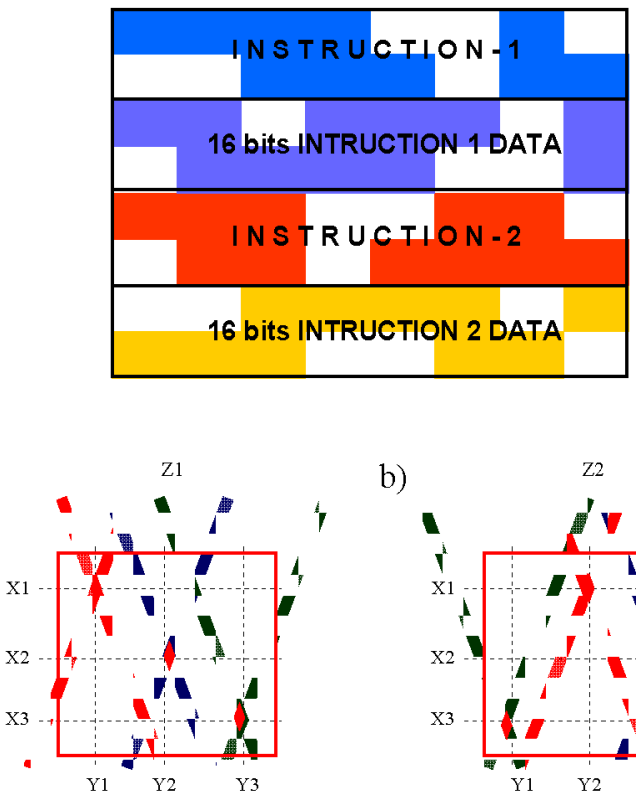

b)
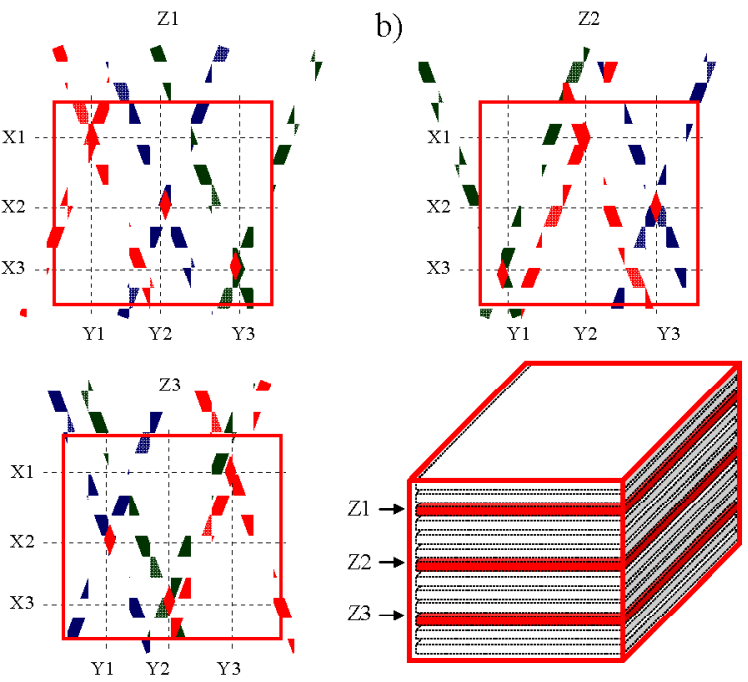

d)

Figure 4. a) Frontal panel of the VI for the 3D position control, b) Distribution of the information into an IBP, c) Frontal panel of the LabVIEW ${ }^{\circledR}$ processing program, d) Experimental localization of the IHF into the IP and the experimental localization of the IP into the crystal.

To execute this program, the user needs to select the other program option. The information processing option starts reading the first IBP, after it moves the crystal to the spatial position of the corresponding IFH. Then it decodes the information and realizes the program instruction codified. All IBP are read and its instructions realized sequentially, 
following the sequence of the program. The frontal panel of this processing program is shown in Fig. 4c. Note that a sensitive control window is displayed on the screen. This control helps us to discriminate between the black and white IPB pixels, at the decodification time. The IFH spatial coordinates must be written in centimeters, and the time delay in milliseconds.

\section{RESULTS.}

The experimental output images for each IBP stored in the photorefractive crystal are shown in table 5 . As we can observe, the experimental images for the IBP were not distorted, although their contrast was diminished. The program sequence was followed by our prototype until the sensitive control was not able to make the distinction between a black and a white IBP pixel. The measurement of the IFH recording time was made after the IBP was displayed in the LCD, whereas the measurement of the information processing time was made by a time control programmed in LabVIEW ${ }^{\circledR}$.

Table 5. Experimental image for each couple of instructions.

\begin{tabular}{|c|c|c|c|c|c|c|c|c|c|c|c|}
\hline INT. & $\mathbf{X}$ & $\mathbf{Y}$ & $\mathbf{Z}$ & WRITE & PROCESS & INT. & $\mathbf{X}$ & $\mathbf{Y}$ & $\mathbf{Z}$ & WRITE & PROCESS \\
\hline $\begin{array}{c}\text { CLA / } \\
\text { LDA } 00\end{array}$ & $\mathrm{X} 1$ & Y1 & $Z 1$ & & & $\begin{array}{l}\text { AND D2 / } \\
\text { SPA }\end{array}$ & $\mathrm{X} 3$ & Y1 & $\mathrm{Z2}$ & & \\
\hline $\begin{array}{l}\text { AND D2 } \\
\text { SPA }\end{array}$ & $\mathrm{X} 2$ & Y2 & Z1 & & & $\begin{array}{l}\text { IOF / } \\
\text { LDA } 03\end{array}$ & $\mathrm{X} 1$ & Y3 & Z3 & & \\
\hline $\begin{array}{c}\text { IOF / } \\
\text { LDA } 01\end{array}$ & X3 & Y3 & $\mathrm{Zl}$ & & & $\begin{array}{c}\mathrm{AND} \mathrm{D2} / \\
\text { SPA }\end{array}$ & $\mathrm{X} 2$ & Y1 & $\mathrm{Z3}$ & & \\
\hline $\begin{array}{l}\text { AND D2 } \\
\text { SPA }\end{array}$ & $\mathrm{X} 1$ & $\mathrm{Y} 2$ & Z2 & & & $\begin{array}{l}\mathrm{IOF} / \\
\mathrm{BUN}\end{array}$ & X3 & $\mathrm{Y} 2$ & Z3 & & Ea \\
\hline $\begin{array}{c}\mathrm{IOF} / \\
\mathrm{LDA} 02\end{array}$ & $\mathrm{X} 2$ & Y3 & $\mathrm{Z} 2$ & & & & $\begin{array}{c}X 1= \\
X 2 \\
X 3=\end{array}$ & 7 & Y3 & 5 & \\
\hline
\end{tabular}

\section{CONCLUSIONS.}

We presented a practical application of a SLHM in an electro-optical setup emulating of an OePDC and its performance was successfully demonstrated in this work. Our system stored the program instructions as IFH in a photorefractive crystal; it read and decodified the IBP of each instruction. It realized all instructions, following the program sequence.

\section{ACKNOWLEDGMENTS.}

Authors thank CONACyT and INAOE for the support provided through the project PY-12822 and the scholarships 190263 and 191110.

\section{REFERENCES.}

1. M. Morris Mano, Computer system architecture, Pearson Education, USA (1994).

2. Barry B. Brey, The Intel microprocessors, Pearson Education, USA (2000).

3. M. Morris Mano, Digital logic and computer design, Prentice Hall, USA (1986).

4. J. W. Goodman, Introduction to Fourier Optics, Third Edition, Ed. Mc Gralw Hill (2000).

5. P. Várhegyi, P. Koppa, F. Ujhelyi, E. Lörincz, Applied Optics, Vol. 44, 3024 (2005). 
Copyright of AIP Conference Proceedings is the property of American Institute of Physics and its content may not be copied or emailed to multiple sites or posted to a listserv without the copyright holder's express written permission. However, users may print, download, or email articles for individual use. 